BNL - 66499

\title{
MEASUREMENT AND OPTIMIZATION OF THE VISA UNDULATOR
}

G. Rakowsky, J. Aspenleiter and L. Solomon

National Synchrotron Light Source

Brookhaven National Laboratory

Upton, New York 11973-5000

R. Carr and R. Ruland

SLAC, Stanford, CA 94309

S. Lidia, LBL, Berkeley, CA 94720

April 1999

National Synchrotron Light Source

Brookhaven National Laboratory

Operated by

Brookhaven Science Associates

Upton, NY 11973

Under Contract with the United States Department of Energy

Contract Number DE-AC02-98CH10886 


\section{DISCLAIMER}

This report was prepared as an account of work sponsored by an agency of the United States Government. Neither the United States Government nor any agency thereof, nor any of their employees, nor any of their contractors, subcontractors or their employees, makes any warranty, express or implied, or assumes any legal liability or responsibility for the accuracy, completeness, or any third party's use or the results of such use of any information, apparatus, product, or process disclosed, or represents that its use would not infringe privately owned rights. Reference herein to any specific commercial product, process, or service by trade name, trademark, manufacturer, or otherwise, does not necessary constitute or imply its endorsement, recommendation, or favoring by the United States Government or any agency thereof or its contractors or subcontractors. The views and opinions of authors expresses herein do not necessarily state to reflect those of the United States Government or any agency thereof. 


\author{
G. Rakowsky ${ }^{+}$, J. Aspenleiter, L Solomon, BNL, Upton, NY 11973 \\ R. Carr, R. Ruland, SLAC, Stanford, CA 94309 \\ S. Lidia, LBL, Berkeley, CA 94720
}

\section{Abstract}

The Visible-Infrared SASE Amplifier (VISA) undulator is an in-vacuum, 4-meter long, $1.8 \mathrm{~cm}$ period, purepermanent magnet device, with a novel, strong focusing, permanent magnet FODO array included within the fixed, $6 \mathrm{~mm}$ undulator gap. The undulator magnet is constructed of 99-cm long segments, joined into a continuous structure. To attain maximum SASE gain requires establishing overlap of electron and photon beams to within 50 microns rms. This imposes challenging tolerances on mechanical fabrication and magnetic field quality, and necessitates use of laser straightness interferometry for calibration and alignment of the magnetic axes of the undulator segments. This paper describes the computerized magnet sorting, pulsed-wire magnetic measurements, trajectory shimming and magnetic axis calibration performed to meet this goal.

\section{INTRODUCTION}

The Visible-Infrared SASE Amplifier (VISA) [1] is a multi-institutional, collaborative experiment whose objective is to demonstrate and analyze Self-Amplified Spontaneous Emission (SASE) from noise to saturation in the $800-600 \mathrm{~nm}$ range. The experiment is being installed at Brookhaven's Accelerator Test Facility (ATF) linac, operating at 70-85 MeV.

Simulations of the VISA FEL [2] have shown that gain is degraded when trajectory wander exceeds $50 \mu \mathrm{m}$ rms. This means that initial trajectory straightness, determination of magnetic centerline, fiducialization and alignment must each be accomplished to within $25 \mu \mathrm{m}$ $\mathrm{rms}$, equivalent to the peak wiggle amplitude at $72 \mathrm{MeV}$. These represent challenging requirements for magnetic and mechanical design, magnetic measurement and metrology. These challenges are addressed in this paper and in companion papers by Libkind [3] and Ruland [4].

\section{UNDULATOR DESIGN}

The VISA undulator has been described in detail previously. [1] It was designed to provide sufficient gain to reach saturation in less than 4 meters, the length available in the ATF beamline. Its compact, modular design is based on a planar, pure-NdFeB, Halbach structure with parameters shown in Table 1.

\footnotetext{
*Work supported in part by U.S. Department of Energy under Contract No. DE-AC02-98CH10886.

+E-mail address: rakowsk1@bnl.gov.
}

Table 1: Undulator parameters

\begin{tabular}{|l|l|}
\hline Period & $18 \mathrm{~mm}$ \\
\hline Gap & $6 \mathrm{~mm}$ (fixed) \\
\hline Block dimensions & $30 \times 10 \times 4.45 \mathrm{~mm}$ \\
\hline Remanence $\mathrm{B}_{r}$ & $1.25 \mathrm{~T}$ \\
\hline Intrinsic coercivity $\mathrm{H}_{c i}$ & $>20 \mathrm{kOe}$ \\
\hline Peak field $\mathrm{B}_{0}$ & $0.75 \mathrm{~T}$ \\
\hline Number of periods & $220(4 \times 55 /$ section) \\
\hline
\end{tabular}

The device is built in four 99-cm long sections, joined into a seamless, 220-period undulator. (Additional sections can be added in the future). Non-steering, displacement-free terminations at each end complete the dipole array.

\subsection{Distributed Strong Focusing}

To attain the needed gain length of $<0.4 \mathrm{~m}$, distributed strong focusing was added to the Halbach array. The compact quadrupole array is formed by pairs of vertically polarized $\mathrm{NdFeB}$ bars, placed lengthwise in the undulator gap, as shown schematically in Figure 1. The proportions were chosen to produce equal vertical and horizontal gradients in the vicinity of the axis. Magnets are mounted in $10-\mathrm{cm}$ long modules, forming a FODO array with $24.75 \mathrm{~cm}$ period, for a total of 16 periods in 4 meters.

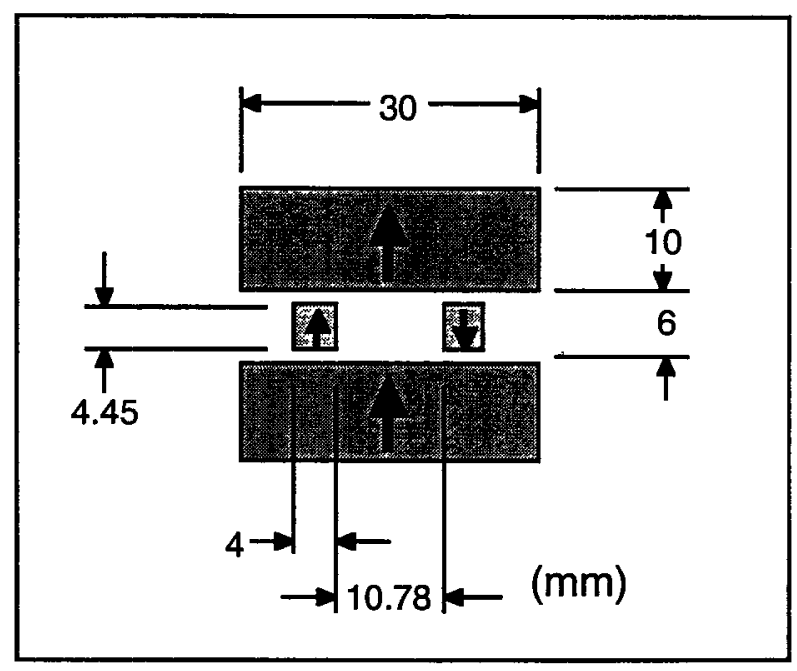

Figure 1. Schematic end view of VISA undulator, showing quadrupole magnets within the undulator gap.

This geometry creates a beam channel only $6 \mathrm{~mm}$ high and about $8 \mathrm{~mm}$ wide between quadrupole holders. An internal vacuum chamber would have reduced the vertical 
aperture even more; leaving too little room for BPM's, diagnostics, or even an alignment laser beam. For this reason the entire undulator was put inside a vacuum vessel. The magnet surfaces facing the beam are lined with $25 \mu \mathrm{m}$ thick nickel foil, for image current continuity.

\section{MAGNET SORTING}

The VISA lattice is comprised of some 1772 magnet blocks forming 220 undulator periods, and 192 blocks forming 16 FODO cells. We specified magnetization tolerances of $1.5 \%$ in magnitude and 1.5 degrees in angle. Left uncorrected, these block errors produce random errors in the field, resulting in excessive beam trajectory walkoff and degradation of FEL interaction. To minimize this adverse accumulation of field errors, the magnetic moments of each magnet block were first measured by the manufacturer. We then used a Monte Carlo algorithm called "threshold acceptance" (similar to simulated annealing) to sort the blocks.[5] A localized 'cost', or 'free energy' function in terms of the moment errors is calculated at each block location along the beamline, then summed to obtain a measure of the level of field error. Starting with a random selection of magnets from the database, the algorithm swaps magnets and recalculates the cost function millions of times, until the total level of field error is reduced to a minimum. In our case, sorting reduced the rms field error by a factor of 14 . The resulting reduction in trajectory walk-off is illustrated in Figure 2.

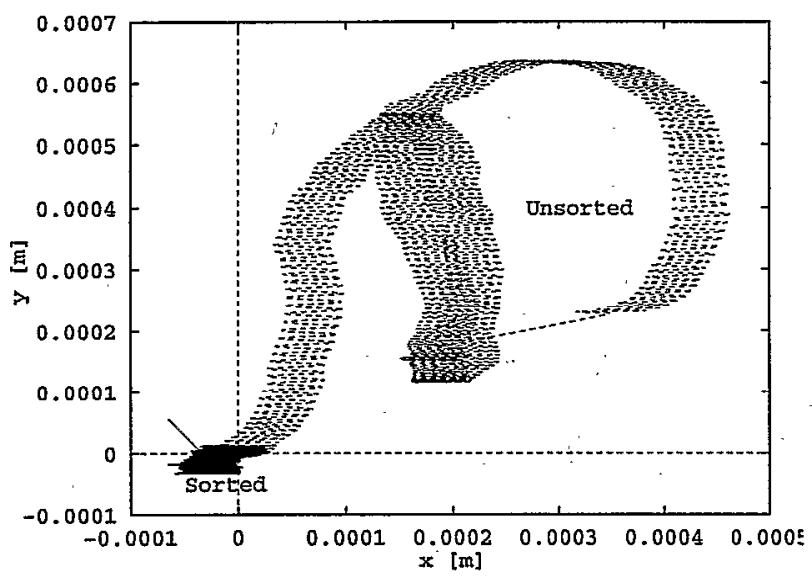

Figure 2: Comparison of undulator trajectory in VISA after sorting vs. a random magnet selection. The view is $y$ vs. $\mathbf{x}$, with the beam axis perpendicular to the page.

For the quadrupole lattice, variations of quadrupole gradient due to errors in the block magnetization only affect betatron motion. However, the quadrupole magnets also contribute dipole errors which cause trajectory errors as well. These errors stem either from magnetization errors or from positioning errors of the quadrupole magnets. Sorting of the quadrupole blocks produced only a very small theoretical improvement in trajectory error. On the other hand, dipole errors due to random quadrupole positioning errors were found to be the dominant cause of trajectory deviation in VISA and became the focus of our measurement and field correction efforts.

\section{PULSED WIRE MEASUREMENT}

The enclosed beam channcl severely limits access by conventional magnetic measurement devices, such as Hall probes. Since we cannot measure field or field errors directly, we rely on magnet block measurement and sorting to assure undulator field quality. To measure and correct trajectory errors, we chose the pulsed wire as the primary measurement tool.[6] Driven by a current step, it provides an instant image of the trajectory, permits rapid, iterative trajectory correction, and is an excellent nullfinding device for locating the quadrupole axis. However, the observed wiggle amplitude in the wire is much smaller, and the betatron wavelength is much longer than that of a $72 \mathrm{MeV}$ electron.

The pulsed wire can also be driven by a current impulse to give an image of angular deviation. However, in this application, the trajectory is the more useful diagnostic.

The pulsed wire technique requires care in setup and interpretation. First, we set up the pulsed wire bench on a massive granite table for stability. Two VISA undulator sections at a time are supported in the center of the table on posts with kinematic supports and $\mathrm{X}$ and $\mathrm{Y}$ adjusters, identical to those used in the final assembly [1]. Smaller granite blocks sit atop the ends of the table, supporting the 4-m long wire, the oil bath vibration damper and the wire position detector. The setup is shown in Figure 3.

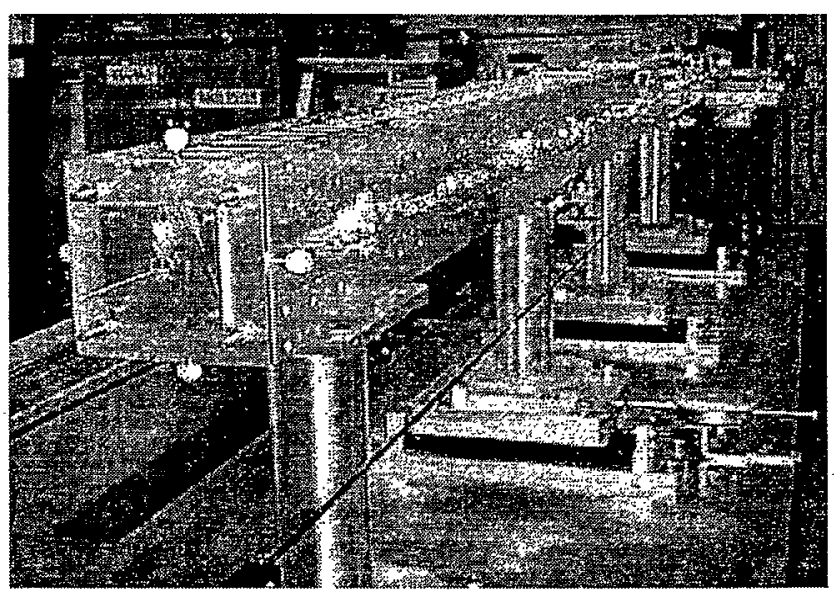

Figure 3: Two VISA sections on the pulsed wire bench.

Despite the stable support structure, the wire tends to vibrate at its resonant frequency $(31 \mathrm{~Hz})$, driven by the electro-magnetic pulses, air currents and environmental sources. To damp the vibrations, one end of the wire is immersed in an oil bath. The $31 \mathrm{~Hz}$ is suppressed by filtering and by the oscilloscope's averaging feature.

Wire sag affects the measurement of the y-component of the trajectory. For this reason the undulator sections were 
designed to be mounted normal or inverted for $\mathrm{x}$-trajectory measurement, or rotated $90^{\circ}$ to measure the y-trajectory in the horizontal plane, independent of wire sag.

Earth's field also contributes a significant error to the trajectory signal. To provide a field-free environment, we added rectangular Helmholtz coils spanning the full length of the bench. The currents needed to cancel Earth's field were determined empirically by observing the trajectory signal without the undulator present.

Thanks to the high gradients and careful setup, we are able to locate the quadrupole centerline in pairs of VISA sections to about $5 \mu \mathrm{m}$ rms.

\section{TRAJECTORY CORRECTION}

Quadrupole misalignment turns out to be the dominant contributor to trajectory error. Despite precisely machined mounting surfaces and the use of a ground spacer bar during installation, random position errors of up to $50 \mu \mathrm{m}$ remained. In a $33 \mathrm{~T} / \mathrm{m}$ gradient, this translates to large steering errors of up to $150 \mathrm{G}-\mathrm{cm}$ per quadrupole module. For this reason the quadrupole holders were mounted on threaded studs accessible from the outside, permitting local $x$-position adjustment of the quadrupole axis. No vertical adjustments were provided, although they would have been desirable. (Cam-type adjusters for vertical positioning are being designed into two additional VISA sections for future expansion.) Instead, y-trajectory corrections were accomplished by strategically clamping discrete NdFeB magnets symmetrically to the sides of the structure.

The undulator sections were also provided with access ports in the upper and lower strongbacks to permit insertion of trim magnet assemblies close to the undulator arrays. The $3 \times 3 \times 2 \mathrm{~mm}$ trim magnets, mounted in sets of four in adjustable holders, are able to provide local steering corrections of up to $30 \mathrm{G}-\mathrm{cm}$ in $\mathrm{x}$ or $\mathrm{y}$.

The magnet sections were first trimmed one at a time. Then they were set up two at a time, to check the trajectory across the joints. Using a combination of quadrupole repositioning, external magnets and the trim magnets, we were able to reduce $x$ and $y$ trajectory errors from several wiggle amplitudes down to a peak-to-peak

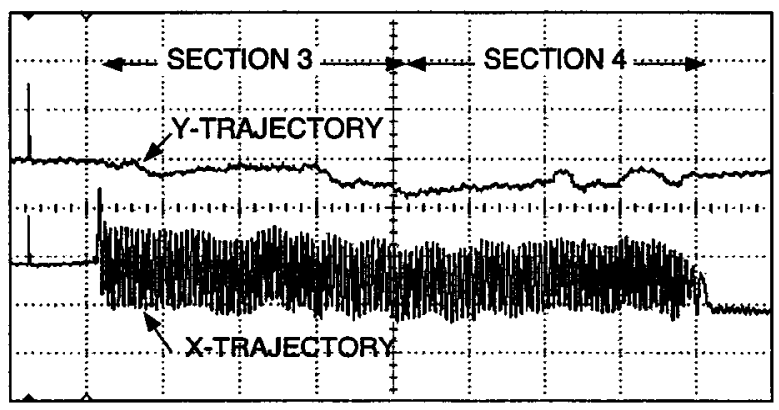

Figure 4: $Y$ (upper trace) and $X$ (lower trace) trajectory in Sections \#3 and \#4 after final trimming. Both have same arbitrary vertical scale. Horizontal scale is $\sim 25 \mathrm{~cm} / \mathrm{div}$. error of less than one wiggle amplitude over 2 meters (or $<10 \mu \mathrm{m} \mathrm{rms}$ ). As an example, Figure 4 shows $x$ and $y$ trajectories in Sections \#3 and \#4, after final trimming.

\section{FIDUCIALIZATION}

To measure position and alignment to better than $25 \mu \mathrm{m}$ in a structure several meters long exceeds the capability of conventional optical survey techniques. We therefore chose laser straightness interferometry, described in detail by Ruland.[4] The interferometer is set up on the bench parallel to the pulsed wire. When the magnetic axis has been aligned to the wire, the straightness interferometer measures the offset of each fiducial from the wire center (i.e., form the magnetic axis). These measurements are done first with the VISA sections in normal orientation, then rotated 90 degrees. This calibrates the magnetic axis relative to the fiducials in both planes. In tests we have demonstrated fiducialization accuracy to $<10 \mu \mathrm{m}$.

During installation of the undulator in the vacuum vessel at the ATF, two straightness interferometers, one in the $x-z$ plane, the other in the $y-z$ plane, will be used to align the undulator sections to the beamline reference laser by reproducing the $\mathrm{x}$ and $\mathrm{y}$ offsets measured on the bench. The expected accuracy of this operation should also be in the $10 \mu \mathrm{m}$ range.

\section{CONCLUSIONS}

We have constructed four 1-m long, strong-focusing undulator sections, located their magnetic axes and trimmed the peak-to-peak trajectory deviations to less than one wiggle amplitude, using the pulsed wire technique. We can locate the magnetic axis of each section, measure fiducial offsets and, it is hoped, align the VISA sections to achieve a final trajectory error well below the required $50 \mu \mathrm{m}$ rms.

\section{ACKNOWLEDGEMENTS}

The authors thank Michael Lehecka (NSLS) for assistance in magnetic measurements, Pedro Frigola (UCLA) for constructing the Earth's field coils, Pietro Musumeci (UCLA) for GENESIS simulations, and all the VISA team members for stimulating discussions and support.

\section{REFERENCES}

[1] R. Carr et al., Proc. 1989 Free Electron Laser Conf.

[2] P. Emma \& H-D. Nuhn, Proc. 1989 Free Electron Laser Conf.

[3] M. Libkind et al., these Proceedings.

[4] R. Ruland et al., these Proceedings.

[5] S.Lidia and R.Carr, Rev. Sci. Inst. 66 (1995) p.1865.

[6] R. Warren \& C. Fortgang, Nucl. Inst. \& Methods A341 (1994) p.444. 
